\title{
LOS CONTRATOS MASIVOS EN EL TRÁFICO COMERCIAL ECONÓMICO
}

\author{
César Aníbal Fernández Fernández ${ }^{1}$
}

\section{RESUMEN}

En los contratos por adhesión o contratos predispuestos una de las partes tiene la posición de dominio por cuanto predispone, redacta, estipula de manera adelantada el contenido del contrato y la otra parte sólo está en la posición alternativa de aceptar o rechazar íntegramente las estipulaciones fijadas declarando su voluntad de aceptar o no. Por su parte, las cláusulas generales de contratación son aquellas redactadas en forma previa y unilateral por una persona 0 entidad, en forma general y abstracta, con el objeto de fijar el contenido normativo de una serie indefinida de futuros contratos particulares con elementos propios, vale decir, son cláusulas contractuales, (por cuanto son elaboradas con la finalidad de ser integradas a un número indefinido de contratos que se celebrarán en el futuro); predispuestas (al haber sido redactadas en forma previa, unilateral de manera general y abstracta con la finalidad de uniformizar el contenido de futuros contratos -pluralidad de negocios-que se celebren en base a dichas cláusulas generales). Si bien es cierto esta situación podría considerarse un tanto injusta por cuanto generan una parte "débil" en la relación jurídica que debe ser protegida, generalmente conocida como "la parte consumidora", lo cierto y verdadero es que estos contratos "masivos" (que vinculan a proveedores y consumidores) son necesarios e indispensables en las sociedades modernas por cuanto reducen los costos de transacción, vale decir, reducen tiempo, dinero y esfuerzo, que genera la contratación, pero también es cierto que exponen a la parte consumidora a estar en una situación de desventaja al limitarse de forma alguna sus derechos, es por ello, la necesidad que el Estado cumpla su rol al determinar los correctos y adecuados mecanismos de control con la finalidad de evitar que los derechos de los consumidores sean vulnerados.

\section{PALABRAS CLAVE}

Contratos por Adhesión o contratos predispuestos. Cláusulas Generales de Contratación. Contratos masivos. Tráfico comercial económico. Cláusulas abusivas. Protección al consumidor.

\begin{abstract}
In adhesion contracts or pre-formulated standard contracts, one party has the dominant position as it is vested with all the bargaining power, the power to draft and insert the contract stipulations in advance while the other party may only acquiesce or may acquiesce or refuse to acquiesce to all the terms of the agreement as proposed. On the other hand, the General Conditions of Contract are drafted in advance by one of the parties, whether an individual or an entity, setting general and abstract performance standards to be applicable to an indefinite number of future contracts which will also contain some specific elements. In other words, such provisions are actually contractual clauses, (because they are made in order to be included in an indefinite number of contracts to be concluded in the future); predetermined (since they have been drafted previously, unilaterally, in general and abstract terms, in order to standardize the content of future contracts - multiple transactions - to be concluded on the basis of such general terms). While this could be regarded as somewhat unfair because one of the parties to the legal relationship would be in a "weak" position and, hence, would require protection -generally known as "consumer protection," it is true that these "mass" contracts (binding suppliers and consumers) are essential in modern societies because they reduce transaction costs, i.e., reduce time, money, and effort to close agreements. Moreover, it is also true that consumers are at a disadvantage since their rights are limited. This is why the State needs to perform its role of determining the correct and appropriate control mechanisms in order to avoid infringement of consumer rights.
\end{abstract}

\section{KEY WORDS}

Adhesion contract (contract of adhesion) or pre-formulated standard contract. General Conditions of Contract. Commercial transactions. Unfair Contract Terms. Consumer Protection.

1 Doctor en Derecho, egresado del Doctorado de la UPSMP. PhD Graduate Doctor oh Philososhy in Business Administration por la Atlantic International University of Miami-Florida. Magíster en Derecho Civil; Maestría en Derecho de la Empresa PUCP. Maestría en Comercio Internacional por la Escuela de Negocios de la Universidad San Pablo CEU- Madrid- España (en convenio con el Instituto Europeo de Posgrado de Madrid-España). Profesor Asociado UNIFE. 


\section{INTRODUCCIÓN}

El artículo 1351 del Código Civil define al contrato como " $\mathrm{El}$ acuerdo de dos o más partes para crear, regular, modificar o extinguir una relación jurídica patrimonial". El contrato "...Es el centro de la vida de los negocios, cuya función principal consiste en satisfacer los intereses inicialmente opuestos, o por lo menos no coincidentes de las partes contratantes. Gracias al contrato y a través de la satisfacción de dichos intereses, cada contratante incrementa su utilidad y de manera indirecta se logra que la sociedad en su conjunto también se beneficie ${ }^{2}$. Asimismo, efectuando un análisis económico: "El contrato entendido como unidad básica de intercambio, es el sinónimo legal del concepto económico de transacción. En este sentido el sistema contractual puede ser entendido como una parte del sistema de mercado, donde el intercambio es el momento esencial de la actividad económica moderna, pues por dicha vía se logra, en teoría, una correcta asignación de los recursos escasos" 3

Por tanto, el contrato en los últimos años es concebido como un elemento esencial para el desarrollo de la economía, la cual se caracteriza por la masificación en la producción de bienes y servicios y por tanto se busca una forma de contratación masiva, en serie, rápida y eficiente; es por ello que surgen los llamados "contratos masivos o en masa", con las cuales se pretende agilizar la celebración de numerosos contratos de tal manera que ello no implique dejar de lado el consentimiento que es el elemento esencial para la existencia y validez de un contrato.

Hoy en día la contratación masiva, obedece a las necesidades del tráfico comercial económico, vale decir, que la producción en serie o en masa de bienes y servicios, se viabilice a través de un instrumento jurídico que permita la celebración inmediata de numerosos contratos, los denominados contratos "masivos" (que vinculan a proveedores y consumidores) que son necesarios e indispensables en las sociedades modernas por cuanto reducen los costos de transacción, es decir, reducen tiempo, dinero y esfuerzo, que genera la contratación.

No obstante que esta modalidad contractual ha sido reiteradamente criticada por cuanto adolecen de la denominada "capacidad de negociación" de una de las partes del contrato, (el consumidor o parte débil) y por ende nos encontramos ante contratos que son predispuestos unilateralmente, es innegable su gran importancia y la función trascendental que cumplen en el derecho contractual y el tráfico comercial económico.

Por tanto, la contratación masiva es un fenómeno jurídico que se ha constituido en un nuevo sistema de contratación privada y que ha surgido ante la necesidad de agilizar el intercambio masivo de bienes y servicios a un menor costo contractual, para lo cual, la etapa de la negociación ha quedado reducida o en la gran mayoría de casos, suprimida ${ }^{4}$.

Agrega el citado jurista que la contratación masiva constituye un "sistema de contratación", en el cual el contenido del contrato ya no es el producto, el resultado del acuerdo conjunto de las partes, sino que éste es predispuesto total o parcialmente por una sola de ellas con antelación a su celebración. Y en este sentido, hoy en día esta forma de contratación se ha convertido en la regla, desplazando a la contratación negociada que ha pasado a constituirse en un medio de contratación excepcional.

Queda claro entonces, que la contratación masiva es una modalidad contractual que hace viable la unión o enlace de dos factores que se juntan en el mercado: por un lado tenemos la producción masiva de bienes y servicios, y del otro, el consumo masivo, confiriéndoles a ambos, un vínculo jurídico obligacional.

De este modo, la contratación masiva cumple principalmente tres funciones; la primera es de carácter jurídico, porque permite el tráfico masivo de intercambio de bienes y servicios; la segunda de carácter social,

2 Messineo Francesco, Doctrina General del Contrato, traducciones de R.O. Fontana - rrosa, S. Sentis Melendo y M. Volterra, Ediciones jurídicas Europa América, Buenos Aires, 1986, T.I, P. 34.

3 Bullard Gonzáles, Alfredo, "Contrato e intercambio económico", en derecho y economía. El análisis económico de las instituciones legales, 2da. Ed., Palestra, Lima, 2006, pp 267-268.

4 De La Puente y Lavalle, Manuel, “El contrato en general”, Fondo Editorial de la Pontificia Universidad Católica del Perú, Lima, 1993, t. III, pág. 71. 
por cuanto al constituirse en el medio que permite la satisfacción de necesidades e intereses, logra el acceso al intercambio de bienes y servicios de una cantidad masiva de personas; y la tercera y última de carácter económico, al agilizar el citado intercambio de bienes y servicios reduciendo los costos que genera dicha transacción.

\section{CONTRATOS POR ADHESIÓN}

En el derecho norteamericano se define a los contratos por adhesión, "Adhesión contracts", como contratos estándar que las empresas ofrecen a los consumidores de bienes y servicios unilateralmente "take it or leave it", "lo tomas o lo dejas", sin otorgarles el mínimo poder de negociación. En consecuencia, se imponen de modo abusivo, por lo general, al consumidor, que por ocupar una posición más débil, en la contratación no pueden negociar sus términos esenciales ${ }^{5}$.

Cabe precisar, que según López Santa María en su obra "Los Contratos: Parte General", la expresión contratos de adhesión proviene de la doctrina francesa, utilizada por primera vez por SALEILLES quien en su De la déclaration de la volonté, la definió como aquellos contratos "en los cuales hay predominio exclusivo de una sola voluntad que actúa como voluntad unilateral, que dicta su ley no ya a un individuo, sino que a una colectividad indeterminada, obligándose por anticipado, unilateralmente, a la espera de la adhesión de los que querrán aceptar la ley del contrato, apoderándose de este compromiso ya creado sobre él mismo".

Para Lacruz Berdejo, el contrato de adhesión es aquel que se propone a la aceptación de una gran masa de público, fijando el proponente las condiciones mediante una formula que imponen a los posibles aceptantes, de modo que a estos no les queda sino aceptar o rechazar el modelo que se les ofrece, y a veces ni eso. Así, el contrato de transporte que celebra el usuario con la compañía de ferrocarriles o el de suministro con la de gas o electricidad es un contrato reglamentado y a la vez, uno de adhesión ${ }^{6}$.

Por tanto, existen diversos conceptos de lo que es un contrato por adhesión, pero veremos que todos son muy similares, así tenemos a la Corporación Nacional de Consumidores y Usuarios de Chile (Conadecus), que define al contrato por adhesión como "aquel cuyas cláusulas han sido propuestas unilateralmente por el proveedor sin que el consumidor, para celebrarlo pueda alterar su contenido"7.

Así mismo, entre los exponentes colombianos encontramos a Laguado Giraldo, quien nos dice que un contrato por adhesión "es aquel acuerdo de voluntades en el cual una de las partes, que generalmente tiene un poder de negociación superior, establece un contenido prefijado para todos los contratos de un determinado tipo que en el ejercicio de la empresa se realicen, de modo tal que la otra, no puede sino acogerse a su clausulado o prescindir de contratar".

Así mismo, el jurista Manuel De La Puente y Lavalle denomina como contrato por adhesión a "aquellos en que las condiciones del mismo son prerredactadas unilateralmente por una de las partes, de manera que la otra solo sólo puede aceptarlas o rechazarlas en bloque"

Los conceptos de contrato por adhesión son todos muy similares, pero también tenemos que en México, Arce Gargollo nos dice que el contrato por adhesión es aquel en el que "una de las partes que generalmente es un empresario mercantil o industrial que realiza una contratación en masa, establece un contenido prefijado para todos los contratos de un determinado tipo, que en el ejercicio en masa se realicen. Las cláusulas de adhesión no pueden ser más que pura y simplemente aceptadas" 10 . Esta definición no resulta muy acertada, pues asevera que las cláusulas del contrato solo pueden ser pura y simplemen-

Black's Law Dictionary, Minn, 1983.

J. L. Lacruz Berdejo: “Derecho de obligaciones”. Vol. II. Barcelona 1987.

Conadecus (2011). Los contratos de adhesión. Recuperado el 27 de septiembre de 2013, de http: / /www.conadecus.cl/ conadecus/?p380.

8 LAGUADO GIRALDO, C. A. (2003). Condiciones generales, cláusulas abusivas y el principio de buena fe en el contrato de seguro. Revista Universitaria: Pontificia Universidad Javeriana, № 105, 231-251.

9 DE LA PUENTE Y LAVALLE, M. (1983). Estudios sobre el contrato privado. Lima: Cultual Cuzco S.A. Editores.

101 ARCE GARGOLLO, J. (2000). Contratos mercantiles atípicos. México: Editorial Porrúa. 
te aceptadas pero como hemos visto existe la posibilidad de que el contrato sea rechazado en su totalidad.

El artículo 1390 de nuestro Código Civil estipula que: "El contrato es por adhesión cuando una de las partes, colocada en la alternativa de aceptar o rechazar íntegramente las estipulaciones fijadas por la otra parte, declara su voluntad de aceptar."

De manera que podemos concluir que en un contrato por adhesión el oferente, con un mayor poder de negociación, establece unilateralmente las condiciones o cláusulas del contrato; mientras que el destinatario solo tiene la posibilidad de aceptar o rechazar el contrato en su totalidad.

Para Diez Picazo y Gullón Ballesteros deben diferenciarse dos fenómenos ${ }^{11}$. El primero se produce cuando todas las cláusulas han sido puestos de conocimientos de los interesados en el momento en que estos dan su conformidad (es la "letra chica" de los contratos de seguros y de suministro de electricidad). El segundo se da cuando han quedado fuera del contrato y el contratante parece adherirse a ella (cuando se compra un boleto de transporte público no se nos dice cuales son las condiciones del contrato que celebramos). Esta distinción ha permitido doctrinalmente separar lo que son contratos de adhesión (los primeros) y lo que son condiciones generales de la contratación (las segundas), entendiendo por condiciones generales del contrato o condiciones generales de la contratación, el conjunto de reglas establecidos por una empresa o grupo de empresas, constitutivas del contenido total o parcial de los contratos que han de celebrar en el desarrollo de sus actividades.

\section{Características}

En Venezuela, el Tribunal Supremo de Justicia considera que son características esenciales de un contrato por adhesión, las siguientes:

1) la oferta tiene un carácter general y permanente, dirigida a persona indeterminada y siendo mantenida por tiempo ilimitado;

2) la oferta generalmente emana de un contratante dotado de cierto poder económico, bien sea originado por sus propias fuerzas o como consecuencia de la unión con otras empresas análogas;

3) el objeto del contrato es la prestación de un servicio privado, pretendido por un sector privilegiado de la comunidad y que solamente la persona jurídica puede proporcionar;

4) la oferta puede aparecer bajo la forma de un contrato tipo o formato cuyas condiciones generales se presentan en bloque a los futuros adherentes particulares; $y$

5) el contrato comprende una serie de cláusulas establecidas generalmente en inte- rés del oferente y en pequeña monta a favor del futuro adherente particular"12.

En cambio, vemos que el maestro Manuel De La Puente y Lavalle $^{13}$ no está de acuerdo con las características establecidas previamente, señalando que un contrato por adhesión no estará necesariamente dirigido a una multiplicidad de personas, sino que pueden plantearse contratos por adhesión con una sola persona o con un grupo de personas determinadas. Por lo cual él solo considera dos elementos típicos de los contratos por adhesión que lo distinguen de los demás, estos son: "El primer elemento que tipifica el contrato por adhesión es que una de las partes fija unilateralmente las estipulaciones contractuales, sin participación de la otra (...)Por otro lado, en ese contrato la aceptación íntegra de las estipulaciones determina la celebración del mismo, en el sentido de que no cabe distinguir entre estipulaciones y oferta, desde que no hay parte del contenido contractual que escape a la fijación unilateral"14.

Cabe precisar que desde una perspectiva de orden económico, Rackoff Todd, en su obra "Contracts of Adhesion: An Essay in Reconstruction", refiere que nos encontramos frente a un contrato por adhesión cuando se satisfacen las siguientes condiciones:

(1) el documento cuya validez legal está en entredicho es una forma impresa que

11 L. Diez - Picazo y A. Gullón Ballesteros. “Sistema de Derecho Civil”. Vol. II. Madrid, 1990.

12 Tribunal Superior de Justicia (2003). Casación Civil. Recuperado el 27 de septiembre de 2013, de http: / /www.tsj.gov.ve/ decisiones/scc/Noviembre/RC-00660-071103-01213.htm

13 DE LA PUENTE Y LAVALLE, M. (1983) Estudios sobre el contrato privado. Lima: Cultural Cuzco S.A. Editores.

14 DE LA PUENTE Y LAVALLE, M. (1993) El contrato en general. Tomo III. Lima: Fondo Editorial de la PUCP. 
contiene una pluralidad de términos y aspira a ser un contrato;

(2) dicha forma ha sido redactada por, o a nombre de, una de las partes de la transacción;

(3) aquella parte que ha redactado los términos participa en numerosas transacciones de aquellas representadas por la forma y realiza rutinariamente este tipo de transacciones;

(4) la forma impresa es presentada al adherente con la prevención que, salvo algunos pocos términos -y las más de las veces ni siquiera esto- podrán ser modificados, manteniéndose el grueso de la estructura del contrato como la diseñó la parte redactora;

(5) después de que las partes -en caso que los hayan- lleguen a un acuerdo sobre aquellos términos abiertos a la negociación, la parte adherente debe firmar la forma;

(6) comparada con la parte adherente, la parte redactora lleva a cabo un número reducido de estas transacciones y;

(7) la principal obligación de la parte adherente en la transacción considerada como un todo es el pago de una suma de dinero.

Según García Amigo, en su obra "Condiciones Generales de los Contratos", los contratos por adhesión podrían sumar un beneficio para los consumidores. Las condiciones generales de los contratos por adhesión evitan la discriminación de los consumidores según sus necesidades, habilidades negociales, etc. Este tipo de negocios sitúan a cada uno de los contratantes en una posición de igualdad respecto de la empresa predisponente. Así "No habría la posibilidad de que un contratante menos avisado pudiera obtener unas condiciones contractuales más desfavorables que otro más sagaz".

De otro lado, Ronald Coase a través de dos artículos La naturaleza de la empresa y El problema del costo social (ambos en COASE, R. La empresa, el mercado y la ley. Alianza Editorial. Madrid: 1994), se refirió a los costos que implica celebrar un contrato. Los costos que identificó Coase se conocen como costos de transacción y pueden agruparse en costos de identificación (consistentes básicamente en localizar al sujeto con quien se debe negociar la titularidad del producto); costos de negociación (es decir, determinar la estructura del vínculo a través del cual se realiza la transferencia) $y$, finalmente, costos de ejecución (aquellos relacionados con la puesta en marcha y realización del acuerdo). Así mismo, los costos de negociación pueden dividirse entre aquellos relacionados con el establecimiento de los valores de amenaza; la determinación del excedente cooperativo y los términos en que se distribuirá el excedente de la cooperación.

\section{Modalidades}

Podemos sostener que se presentan dos modalidades de contratos por adhesión: los contratos por adhesión de libre decisión y los contratos por adhesión de estado de necesidad.

Dentro de los referidos en primer lugar, vale decir, en los contratos por adhesión de libre decisión se encuentran la gran mayoría de contratos masivos, donde el destinatario tiene la amplia libertad de elegir si acepta o no adherirse al contrato que se le propone. Tiene un abanico de posibilidades entre los diversos oferentes $u$ ofertantes de bienes y servicios y se sobreentiende que se encuentra debidamente informado de los aspectos a favor o contra que le implican la celebración del contrato. Citamos como ejemplo los casos que una persona desee adquirir un electrodoméstico, o cualquier bien, puede sin duda, adquirirlo en cualquier tienda por departamentos o si se trata de un celular tenemos hasta tres operadores de servicios de telefonía móvil. Queda claro, que elegirá el que más le convenga en razón de precio, calidad, etc, etc.

En cambio, en los contratos por adhesión de estado de necesidad la cosa es distinta. El destinatario no tiene otra opción que adherirse al contrato por cuanto existe un solo operador del servicio. No le queda otra alternativa que adherirse al contrato para lograr satisfacer una necesidad, que es de primer orden, como por ejemplo el contrato de suministro de agua, de luz, entre otros.

\section{ConTroversia}

Existe una controversia, con respecto a si el contrato por adhesión es en realidad un contrato o no, (ello por cuanto como bien sabemos los contratos tienen tres fases bien marcadas: a) La negociación; b) la celebración y c) la ejecución; y es precisamente la primera de las nom- 
bradas -capacidad negocial- la cual adolecen los contratos por Adhesión) entre civilistas o contractualistas, entre ellos Planiol y Ripert, Josserand, Domat, Lafaille, Salvat, y publicistas o autores de derecho público, entre los que encontramos a Leon Duguit y Mauricio Hauriou. Desde el punto de vista de los publicistas López Argáez nos dice, comentando a Duguit que el contrato de adhesión "no es tal contrato, en vista que uno de los contratantes, el oferente, está emitiendo una voluntad reglamentaria; el otro se encuentra en la imposibilidad de discutir los términos del contrato y acepta las condiciones preestablecidas o no hay tal operación jurídica, cuyo resultado dice el jurista, no es obra de dos voluntades"15, así podemos ver que los publicistas consideran que en los llamados contratos por adhesión solo existe una voluntad, por lo cual no debería considerársele un contrato, sino, más bien, un acto jurídico.

Por otro lado, para los civilistas o contractualistas sí estiman que se le considere un contrato pues "los términos del contrato, las estipulaciones, etc., han sido obra de una voluntad; mientras el contrato no se formula (...) En ese momento hay una simple oferta, no hay contrato, la parte ofertante $o$ adherente lo lee $y$ manifiesta su voluntad y acepta, ya se constituye el contrato (...) $\mathrm{Si}$ alguno de los contratantes no ha leído el contrato, habrá dolo, error, vicio de la voluntad en su consentimiento, podría alegar la nulidad del contrato, absoluta o relativa, según la naturaleza del vicio; pero habrá contrato; no será válido, pero tal situación no desvirtúa la naturaleza misma contractual de esa operación jurídica" ${ }^{16}$. Compartimos esta última opinión.

\section{ESTIPULACIONES INVÁLIDAS EN LOS CONTRATOS DE ADHESIÓN}

El artículo 1398 del Código Civil estipula que en los contratos por adhesión "no son válidas las estipulaciones que establezcan, en favor de quien las ha redactado, exoneraciones o limitaciones de responsabilidad; facultades de suspender la ejecución del contrato, de rescindirlo o de resolverlo; y de prohibir a la otra parte el derecho de oponer excepciones o de prorrogar o renovar tácitamente el contrato".

\section{Cláusulas generales de CONTRATACIÓN}

Según el artículo 1392 del Código Civil Peruano define a las cláusulas generales de contratación como aquellas redactadas previa y unilateralmente por una persona o entidad en forma general y abstracta, con el objeto de fijar el contenido normativo de futuros contratos, con elementos propios de ellos.

El maestro sanmarquino Max Arias-Schreiber, precisa que las cláusulas generales de contratación “...son formuladas de manera preventiva y unilateral por una persona o un grupo de personas, que fijan de un modo abstracto el contenido normativo general o accesorio de una serie de contratos individuales, de manera que éstos se ajustan a aquéllas. En suma, se trata de fórmulas abstractas y generales, de allí su nombre, que se incorporan a los contratos individuales y existe en ellas, por consiguiente, un fenómeno de intercalación. Desde luego, estas cláusulas sólo son vinculantes en la medida que se celebran los contratos individuales". ${ }^{17}$

Como conjunto de cláusulas tienen entonces la finalidad de fijar las condiciones para un sinnúmero de futuros contratos, los mismos que contarán además con sus propios elementos característicos. Respecto a estas cláusulas la exposición de motivos del Código Civil, que recogió la opinión del ponente, Dr. Max Arias Schreiber, señala que: "...son formuladas de manera preventiva y unilateral por una persona o grupo de personas, que fijan de un modo abstracto el contenido normativo general o accesorio de una serie de contratos individuales, de manera que éstos se ajustan a aquéllas. En suma, se trata de fórmulas abstractas y generales, de donde su nombre, que se incorporan a los contratos individuales y existe en ellas, por consiguiente, un fenómeno de intercalación. Desde luego estas cláusulas sólo son vinculantes en la medida que se celebran los contratos individuales" ${ }^{\prime 18}$.

Las cláusulas generales provocan una reducción de los costos de celebración y una regulación de los contratos. Así mismo fa-

5 LÓPEZ ARGÁEZ, J. R. La Controversia del Contrato de Adhesión. Tohil: Revista Jurídica, 21-22.

LÓPEZ ARGÁEZ, Op. Cit.

ARIAS SCHREIBER PEZET, Max; Exégesis del Código Civil de 1984; $2^{\text {a }}$ Edic.; Gaceta Jurídica S.A. Lima, $2000 ;$ Tomo I; p. 160.

18 REVOREDO DE DEBAKEY, Delia; Código Civil, Exposición de Motivos y Comentarios; Edit. Okura; Lima 1985; vol. Vl; p. 54 
cilitan la coordinación, y hacen posible el cálculo anticipado del costo de producción de los bienes y servicios que ofrece la empresa.

Arias Schereiber señala como sus elementos: la redacción previa, el carácter unilateral, su carácter preventivo y abstracto, constituida de elementos propios y comunes ${ }^{19}$, su aceptación en bloque (el Código no lo dice y es un defecto del mismo) y su inmutabilidad.

En cuanto a su naturaleza jurídica encontramos que existen dos posiciones: La primera, establece que las cláusulas generales de contratación tienen carácter normativo. La segunda, precisa que en las cláusulas generales existe un simple elemento integrante de los contratos, sometidos, por tanto, a las leyes.

\section{Cláusulas Generales como Re-} glas Normativas: ${ }^{20}$ Consideran las condiciones como obligatorias, tanto para el prerredactante como para quien se adhiere a ellas por ser impuestas, dado su carácter normativo y no por ser el resultado de un común acuerdo. La argumentación de los autores de esta primera posición se resume de la siguiente manera: Las condiciones generales son indiscutiblemente obligatorias, porque suplen los vacíos de la ley en el área de contratos. En tal sentido, estas cláusulas unilateralmente impuestas por las empresas a los consumidores merecen ser calificadas como una fuente del Derecho Comercial, por cuanto se aplican sin ser sometidas a la voluntad de los contratantes.

Cláusulas Generales como Declaración Contractual: Es una posición que les otorga el carácter de contractuales. Dentro de ésta, a su vez, es posible distinguir hasta tres posturas diferentes:

$1^{\circ}$ Que advierte en las cláusulas generales la regulación de la relación vinculatoria entre las partes, no por tener eficacia jurídica por sí misma, sino porque representan una oferta incondicionalmente aceptada.

$2^{\circ}$ Según la cual jurídicamente la fuerza de las cláusulas generales se deriva del acuerdo de voluntades de las partes, pero que necesitan indispensablemente de la adhesión para poder establecer el vínculo entre ellas.

$3^{\circ}$ Se pronuncia en el sentido que las cláusulas generales pueden formar parte del contrato individual, aún cuando el adherente no las conozca, siempre que hubiera podido conocerlas a través de una diligencia ordinaria o usual, antes de celebrar el contrato particular.

Los principios de UNIDROIT definen las cláusulas "estándar" como aquellas preparadas con antelación por una de las partes para su uso general, repetido y utilizadas, de hecho, sin ser negociadas por la otra parte. ${ }^{21}$

Coincidimos con Mosset Iturras- pe cuando señala que las cláusulas generales de contratación, son un mecanismo ajustado a nuestra realidad inmersa en la "sociedad de consumo" que obliga a una mayor celeridad en las transacciones comerciales. Por ello el Derecho se ve también obligado a adecuarse, y lo hace regulando. Empero, Derecho y Economía no pueden desconocer que las relaciones económicas del Mercado, tienen un "orden natural", una impronta, que se orienta a la eficiencia, al lucro, y a la productividad. $Y$ las "relaciones económicas del Mercado" no pueden dar la espalda al contenido ético del Derecho que está dado por el respeto a la dignidad de la persona humana, cualquiera sea ella. ${ }^{22}$

\section{LAS CLAÚSULAS ABUSIVAS $Y$ LA PROTECCIÓN AL CONSUMIDOR}

El Código de Protección y Defensa del Consumidor, en el inciso 1 del Artículo 49, nos dice sobre las cláusulas abusivas que, "en los contratos por adhesión y en las cláusulas generales de contratación no aprobadas administrativamente, se consideran cláusulas abusivas y, por tanto, inexigibles todas aquellas estipulaciones no negociadas individualmente que, en contra de las exigencias de la buena fe, coloquen al consumidor, en su perjuicio, en una situación de desventaja o desigualdad o anulen sus derechos"23.

Como ya hemos visto, en un contrato por adhesión siempre existirá una parte con el poder

19 ARIAS SCHREIBER, MAX. (2000). Contratos celebrados por adhesión y cláusulas generales de contratación. Lima: Gaceta Jurídica S.A.

20 Posición sostenida por la parte de la doctrina alemana. Defendida en la actualidad por Joaquín Garrigues.

21 Obtenido de http://www.unidroit.org

22 MOSSET ITURRASPE, J. (1997). Principio de la buena fe y las cláusulas contractuales abusivas. Arequipa.

23 Ley № 29571 Código de Protección y Defensa del Consumidor (2010). Diario Oficial El Peruano . 
para fijar o redactar las estipulaciones del contrato, por lo cual tendría ventaja sobre la otra parte, que quedaría en cierto estado de subordinación, por lo cual se deben regular estas relaciones, es por eso que se crea el Código de Protección y Defensa del Consumidor.

El inciso 2 del artículo $V$ del Título Preliminar del Código de Protección y Defensa del Consumidor estipula que:

"En cualquier campo de su actuación, el Estado ejerce una acción tuitiva a favor de los consumidores. En proyección de este principio en caso de duda insalvable en el sentido de las normas o cuando exista duda en los alcances de los contratos por adhesión y los celebrados en base a cláusulas generales de contratación, debe interpretarse en sentido más favorable al consumidor."24

En el Código de Protección y Defensa del Consumidor encontramos que regula los requisitos que deben tener las cláusulas que contiene un contrato de consumo por adhesión en su artículo 48, diciendo:

"En los contratos de consumo celebrados por adhesión o con cláusulas generales de contratación, debe cumplirse con los siguientes requisitos:

a. Concreción, claridad y sencillez en la redacción, con posibilidad de comprensión directa, sin reenvíos a textos o documentos que no se faciliten previa o simultáneamente a la conclusión del contrato, y a los que, en todo caso, debe hacerse referencia expresa en el documento contractual.

b. Accesibilidad y legibilidad, de forma que permita al consumidor y usuario el conocimiento previo del contenido del contrato antes de su suscripción.

c. Buena fe y equilibrio necesario en los derechos y obligaciones de las partes, lo que en todo caso excluye la utilización de cláusulas abusivas. Lo dispuesto en el presente artículo resulta de aplicación a los contratos celebrados en base a cláusulas generales de contratación, se encuentren o no sometidas a aprobación administrativa." 25

\section{ProtecCión AL CONSUMIDOR EN LA LEGISLACIÓN COMPARADA}

EN COLOMBIA: Encontramos la Ley $\mathrm{N}^{\circ} 1480$ Estatuto del Consumidor que establece entre los derechos de los consumidores y usuarios la protección contractual que significa "ser protegido de las cláusulas abusivas en los contratos de adhesión, en los términos de la presente ley"26.

EN ECUADOR: En Ecuador encontramos la Ley Orgánica de Defensa del Consumidor ${ }^{27}$, la cual en su capítulo VII establece artículos para la Protección Contractual, como que el contrato debe ser redactado con caracteres legibles, en términos claros y comprensibles, escritos en castellano; además prohíbe cláusulas que eximan, atenúen o limiten la responsabilidad de los proveedores por vicios en los bienes o servicios prestados; etc.

EN ESPAÑA: Acá encontramos la Ley General para la Protección de los Consumidores y Usuarios $^{28}$, pero acá no hablan de contratos de adhesión, sino de Cláusulas no negociables individualmente, estableciendo los requisitos que debe presentar, su sometimiento a la aprobación y control de las Administraciones públicas, y la obligación de los Notarios y los Registradores de la Propiedad y Mercantiles de informar a los consumidores y usuarios.

\section{Conclusiones}

1.- La contratación masiva es hoy en día necesaria e indispensable para un tráfico comercial económico ágil y fluido. Estos se viabilizan mediante la celebración de contratos por adhesión o sobre la base de cláusulas generales de contratación.

2.- En los contratos por adhesión o contratos predispuestos una de las partes tiene la posición de dominio por cuanto predispone, redacta, estipula de manera adelantada el contenido del contrato y la otra parte sólo está en la posición alternativa de aceptar o rechazar íntegramente las estipulaciones fijadas declarando su voluntad de aceptar o no. Por su parte, las cláusulas generales de contratación son aquellas redactadas

\section{Ibidem.}

Ley № 29571 Código de Protección y Defensa del Consumidor (2010). Diario Oficial El Peruano.

Ley 1480 Estatuto del Consumidor (2011). En Diario Oficial № 48.220. Colombia.

Ley 2000-21 Ley Orgánica de Defensa del Consumidor. En Registro Oficial № 520. Ecuador.

28 Ley General para la Defensa de los Consumidores (2007). En Boletín Oficial № 287 del Estado. Madrid. 
en forma previa y unilateral por una persona o entidad, en forma general y abstracta, con el objeto de fijar el contenido normativo de una serie indefinida de futuros contratos particulares con elementos propios, vale decir, son cláusulas contractuales, (por cuanto son elaboradas con la finalidad de ser integradas a un número indefinido de contratos que se celebrarán en el futuro); predispuestas (al haber sido redactadas en forma previa, unilateral de manera general y abstracta con la finalidad de uniformizar el contenido de futuros contratos -pluralidad de negocios-que se celebren en base a dichas cláusulas generales).

3.- Cabe precisar que el hecho que en la contratación masiva no se de la fase o etapa de la negociación (capacidad negocial de las partes) no implica de manera alguna que deje de ser "un contrato". Por cuanto no obstante que en un momento inicial solo existe la voluntad de una de las partes (oferente), lo que la convertiría en un acto jurídico, posteriormente aparece la otra parte, (el adherente), quien va a manifestar su voluntad de aceptar íntegramente las estipulaciones fijadas brindando su consentimiento.

4.- La contratación masiva permite satisfacer una gran necesidad, alcanzar la rapidez que exige el tráfico contractual masivo, permitiendo reducir los costos de transacción, vale decir, reducen tiempo, dinero y esfuerzo, que genera la contratación, pero también es cierto que exponen a la parte consumidora a estar en una situa- ción de desventaja al limitarse de forma alguna sus derechos, es por ello, la necesidad que el Estado cumpla su rol al determinar los correctos y adecuados mecanismos de control con la finalidad de evitar que los derechos de los consumidores sean vulnerados.

\section{Bibliografía}

ARCE GARGOLLO, J. (2000). Contratos mercantiles atípicos. México: Editorial Porrúa.

CÓDIGO CIVIL PERUANO. (2012). Lima: Gaceta Jurídica.

CONADECUS. (27 de Septiembre de 2013). Conadecus: Asociación de Consumidores.Obtenido de http://www.conadecus.cl/conadecus $/ \mathrm{p}=380$

DE LA PUENTE Y LA VALLE, M. (1983). Estudios sobre el contrato privado. Lima: Cultural Cuzco S.A. Editores.

DE LA PUENTE Y LA VALLE, M. (1993). El contrato en general. Tomo III. Lima: Fondo Editorial de la PUCP.

DE LA PUENTE Y LA VALLE, M. (2000). Cláusulas Generales de Contratación. Lima: Gaceta Jurídica.

ESPINOZA ESPINOZA, J. (1998). Cláusulas Vejatorias en los Contratos Estipulados Unilateralmente. Lima: Fondo Editorial PUCP.

LAGUADO GIRALDO, C. (2003). Condiciones generales, cláusulas abusivas y el principio de buena fe en el contrato de seguro. Revista Universitaria: Pontificia Universidad Javeriana, $\mathrm{N}^{\circ}$ 105, 231-251.

Ley 2000-21 Ley Orgánica de Defensa del Consumidor. En Registro Oficial No 520. Ecuador.

Ley General para la Defensa de los Consumidores (2007). En Boletín Oficial $N^{\circ} 287$ del Estado. Madrid.

Ley No 29571 Código de Protección y Defensa del Consumidor (2010). En Diario Oficial El Peruano. Perú.

Ley 1480 Estatuto del Consumidor (2011). En Diario Oficial $N^{\circ}$ 48.220. Colombia.

LÓPEZ ARGÁEZ, J. R. (s.f.). La Controversia del Contrato de Adhesión. Tohil: Revista Jurídica, 21-22.

ARIAS SCHREIBER, MAX. S. (2000). Contratos Celebrados por Adhesión y Cláusulas Generales de Contratación. Lima: Gaceta Jurídica S.A.

MESSINEO, F. (2001). Manual de Derecho Civil y Comercial. Buenos Aires: Jurídicas Europa.

MOSSET ITURRASPE, J. (1997). Principio de la Buena Fe y las Cláusulas Contractuales Abusivas. Arequipa.

SONCCO MENDOZA, P. (2003). Los contratos por adhesión y las cláusulas generales de contratación. Revista de la Facultad de Derecho de la UNSA, Año 5 Número 5. 